\title{
Controlling Water Vapor in Gas-Cell Microscopy Experiments
}

\author{
K. A. Unocic ${ }^{1}$, H. M. Meyer III ${ }^{1}$, F. S. Walden ${ }^{2}$, N. L. Marthe ${ }^{2}$, W. C. Bigelow ${ }^{3}$ and L. F. Allard ${ }^{1}$ \\ 1. Materials Science \& Technology Div., Oak Ridge National Laboratory, Oak Ridge, TN. \\ 2. Protochips Inc., Morrisville, NC. \\ ${ }^{3 .}$ Department of Materials Science \& Engineering, University of Michigan, Ann Arbor, MI.
}

There is an increasing interest in understanding the role of water vapor on the high-temperature degradation behavior of structural materials when subjected to aggressive environments [1]. For example, thermal barrier coatings are used to protect turbine blades from high-temperature degradation and the presence of water vapor can substantially decrease lifetime [2]. Our aim here, is to develop methods and protocols for introducing and quantifying water vapor in a MEMS-based closed-cell in situ reactor system (Protochips Atmosphere ${ }^{\mathrm{TM}}$ ), for studies focused on the effects of environmental exposure in hightemperature structural materials.

The gas-handling manifold of the Atmosphere system allows computer-controlled mixing of up to three gases in two independent experimental gas tanks, and also control of the reaction work-flow protocols. The primary challenge for incorporating water vapor in the supplied gas is that it is not possible to effectively heat all of the capillary, supply and return gas lines to the reactor holder, so water vapor can only be supplied under normal room temperature conditions. At room temperature, water vapor comprises a maximum of $\sim 2 \%$ of the total gas composition (e.g. $\sim 17$ Torr partial pressure in 1 atmosphere). Thus, water vapor concentrations above $2 \%$ require lower total pressure in the closed-cell reactor; e.g. 17 Torr water vapor partial pressure in 170 Torr total gas pressure would yield $10 \%$ of water vapor in the gas composition.

To introduce water vapor into the supply tank, a new water delivery fixture was fabricated and attached to one of the entry ports on the gas delivery manifold (Figure 1a inset). A small stainless steel vessel is initially charged with 1-2 $\mathrm{ml}$ of water, through a valve $\left(\mathrm{V}_{\mathrm{C}}\right)$ on the end of the vessel. The vessel can be pumped through the gas manifold to a pressure of 17 Torr, and a hand valve $\left(\mathrm{V}_{\mathrm{V}}\right)$ on the manifold side of the vessel can be used to incorporate water vapor into the vacuum tank during the experiment. A supply tank is purged several times with $\mathrm{N}_{2}$ and pumped to $<0.1$ Torr, water vapor is then introduced to $\leq 17$ Torr. Experiments in 100\% water vapor can then be run at this pressure, or additional gas(es) can be added to the Supply tank as needed to give the ultimately desired gas composition.

We first explored the use of cube-shaped $\mathrm{MgO}$ nanocrystals as potential indicators for the presence of water vapor in the cell [3]. However, since the morphological changes on the surface of the $\mathrm{MgO}$ crystals were small, different quantitative measurements of gas composition were necessary to further support and confirm the presence of water vapor during in situ S/TEM testing. Thus, a residual gas analyzer (RGA) from Stanford Research Systems (SRS 100 RGA), equipped with an electron multiplier was mounted on a turbo-pumped station (Pfeiffer Hi-Cube 80 Eco) with a cold-cathode gauge attached, and coupled to a computer-controlled leak valve (Pfeiffer Vacuum, D-35614 Asslar) (Figure 1a). The RGA system is connected to a stainless steel capillary on the return side of the in situ gas-reactor holder. Heating tape around the RGA chamber allows bake-outs between experiments to remove residual water vapor and gases. Gas composition measurements are possible when the RGA chamber pressure is in the $\leq 10^{-5}$ Torr range; an ultra-high vacuum of $<2 \times 10^{-8}$ Torr can ultimately be reached in the chamber alone. The automatic leak valve is controlled via Atmosphere Clarity ${ }^{\mathrm{TM}}$ software, and is responsible for monitoring 
the returning gas into the RGA chamber. A return capillary to the manifold is isolated from the leak valve by a hand valve (HV), so that when the leak valve is closed, all the returning gas is directed to the vacuum tank in the gas manifold (Figure 1b). We can also choose to partially flow the returning gas through the RGA system and the vacuum tank with both valves open. Figure $2 \mathrm{a}$ shows the gas manifold schematic with Tank 1 charged to 16 Torr water vapor. The ability to detect water vapor, atomic mass unit (amu) 18, passing through the gas cell is shown in Figure 2b, where the inset RGA scans show the large visible peak increase at $18 \mathrm{amu}$. Experiments are in progress to calibrate the gas compositions via RGA analysis, so we can ultimately study oxidation reactions on structural materials in the presence of water vapor [4].

References:

[1] M. G. Burke et al, Ultramicroscopy 176 (2017), p. 46

[2] K A Unocic and B A Pint, Surf. \& Coatings Tech. 215 (2013), p. 30.

[3] K A Unocic et al, Microsc. Microanal. 23 (S1) (2017), p. 940.

[4] Research sponsored by the Laboratory Directed Research and Development program, ORNL, managed by

UT-Battelle LLC for the US Department of Energy.
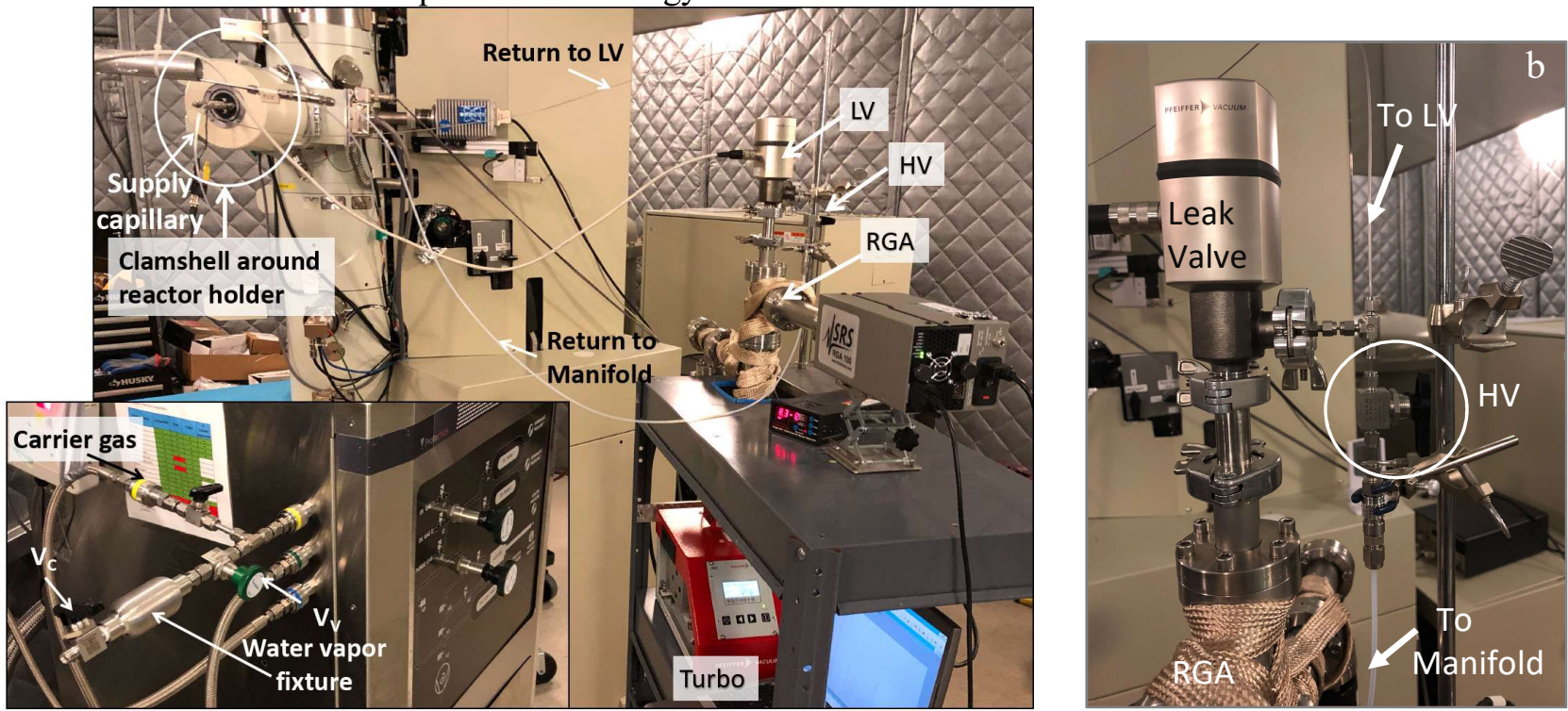

Figure 1. a) RGA connection details to the reactor holder (inside Clamshell). Inset: Arrangement of water vapor delivery fixture on adjacent Atmosphere gas manifold. b) Leak Valve (LV), Hand Valve (HV) and capillary connections.
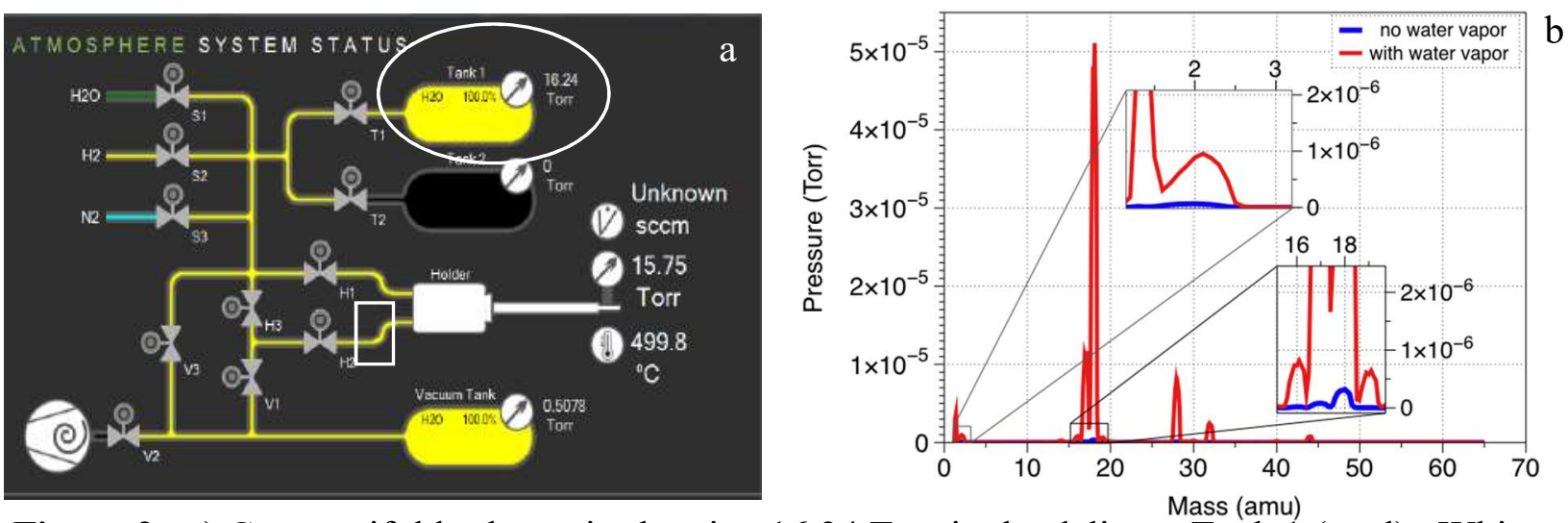

Figure 2. a) Gas manifold schematic showing 16.24 Torr in the delivery Tank 1 (oval). White rectangle locates position of RGA system; b) Detection of water vapor through the gas cell is shown by the RG system before and after introduction of water vapor. 